\title{
Analysis of frequency uncertainty on positioning accuracy based on WINNER II Channel

\author{
Baode $\mathrm{Wu}^{1}, \mathrm{Xin} \mathrm{Shi}^{1}$, and Jiyan Huang ${ }^{2, \mathrm{a}}$
}

${ }^{1}$ North engineering Co.,LTD of the electrification bureau group, crcc, Taiyuan, China

${ }^{2}$ University of Electronic Science and Technology of china (UESTC), Chengdu, China

ahuangjiyan@uestc.edu.cn

Keywords: CRLB, RSS, Geolocation, frequency change, positioning accuracy.

\begin{abstract}
Conventional geolocation techniques include the time of arrival (TOA), the time difference of arrival (TDOA), the angle of arrival (AOA), the received signal strength (RSS) , the fingerprint positioning based methods and etc. Among these location techniques, a method based on RSS has attracted much attention because of its low complexity and cost of devices. Based on channel model obtained from measurements, the positioning accuracy of RSS location method is affected by the frequency. Thus, analysis of the impact of frequency uncertainty is a very important issue for mobile location. Although many performance analyses have been addressed for RSS location method, the impact of frequency uncertainty on positioning accuracy for RSS has not been studied in the literature to the best of our knowledge. In this paper, theoretical analysis and simulations are both provided for analyzing the impact of frequency uncertainty on positioning accuracy.
\end{abstract}

\section{Introduction}

Location information is valuable to a myriad of applications of wireless networks [1]. Several geolocation techniques have been used to estimate mobile station (MS) position including the time-of-arrival (TOA), the time-difference-of-arrival (TDOA), the angle-of-arrival (AOA), the received signal strength (RSS) based methods or hybrid location methods. Among these location techniques, a method based on RSS has attracted much attention because of its low complexity and cost of devices [2,3]. Various RSS based location algorithms and their Cramer-Rao lower bounds (CRLBs) were addressed in the literature. Unfortunately, those studies [2,3] don't consider the frequency uncertainty in a practical system. For real wireless channel, Doppler shift and estimation errors will result in the uncertainty of carrier frequency estimation. WINNER II model is a famous channel model which is built on measurements. It is shown that RSS measurements depend on the estimate of frequency. This implies that the positioning accuracy of RSS location method may be affected by the frequency. Thus, analysis of frequency uncertainty is a very important issue for RSS location technique.

In this paper, theoretical analysis and simulations are both provided for analyzing the impact of frequency uncertainty on positioning accuracy which have not been studied in the literature. It is well known that CRLB provides a benchmark to evaluate the performance of any unbiased estimator and determines the physical impossibility of the variance of an unbiased estimator being less than the bound [4]. This paper evaluates the effects of frequency uncertainty on mobile location in terms of CRLB. Firstly, two CRLBs based WINNER II channel will be derived for the cases with perfect or imperfect frequency estimation. Then the relationship between the proposed CRLBs is given in the paper. Theoretical analysis and simulations show that the effects of the frequency uncertainty on performance of location estimation would be negligible.

\section{WINNER II path loss model}

The basic WINNER II path loss model [5] is briefly introduced in this section. The path loss model is typically in the form of (1), where $d$ is the distance between the transmitter and the receiver in $[\mathrm{m}], f_{c}$ is the carrier frequency in [GHz], the fitting parameter A includes the path-loss 
exponent (PLE), parameter $\mathrm{B}$ is the intercept, parameter $\mathrm{C}$ describes the path loss frequency dependence, and $\mathrm{X}$ is an optional, environment-specific term (e.g., wall attenuation in the A1 NLOS scenario).

$$
\beta=\mathrm{A} \log _{10} d[m]+B+C \log _{10} \frac{f_{c}[G H z]}{5.0}+X+n
$$

where $n$ is the measurement error caused by shadow fading, and it is subject to zero mean Gaussian distribution with variance $\sigma_{d B}^{2}$.

\section{Performance Analysis}

It is well known that the CRLB sets a lower limit for the variance or covariance matrix of any unbiased estimate of unknown parameters [2]. This paper evaluates the effects of frequency uncertainty on mobile location in terms of CRLB. Two CRLBs based WINNER II channel are derived for the cases with perfect or imperfect frequency estimation. Then the relationship between the proposed CRLBs is given in the paper.

Assuming that $(x, y)$ is the position of a MS and $\left(x_{i}, y_{i}\right)$ is the position of the $i$ th BS in a N-BSs system. Denote the measurement with noise of $\{*\}$ as $\left\{\%\right.$. The true distance $d_{i}$ between BS $\mathrm{i}$ and MS can be modelled as $d_{i}^{2}=\left(x_{i}-x\right)^{2}+\left(y_{i}-y\right)^{2}$.

Let $\mathbf{P}=\left[\begin{array}{lll}\beta_{1}^{\prime 0} & \mathrm{~L} & \beta_{N}^{\prime 0}\end{array}\right]^{T}$ be a vector of path loss measurements and $\boldsymbol{\theta}$ is an unknown parameter vector. The CRLB matrix is defined as the inverse of the Fisher information matrix (FIM) $\mathbf{J}$ :

$$
E\left(\left(\boldsymbol{\theta}^{\circ} \boldsymbol{\theta}\right)\left(\boldsymbol{\theta}^{\circ} \boldsymbol{\theta}\right)^{T}\right) \geq \mathbf{J}^{-1}=\left[E\left[\frac{\partial \ln f(\mathbf{P}, \boldsymbol{\theta})}{\partial \boldsymbol{\theta}}\left(\frac{\partial \ln f(\mathbf{P}, \boldsymbol{\theta})}{\partial \boldsymbol{\theta}}\right)^{T}\right]\right]^{-1}
$$

where $\boldsymbol{\theta}$ is an estimate of $\boldsymbol{\theta}, f(\mathbf{P}, \boldsymbol{\theta})$ is joint probability density function (PDF) of $\mathbf{P}$ and $\boldsymbol{\theta}$.

From (1), the probability density function (PDF) of the $i$ th $P_{i}^{\prime \prime}$ can be written as:

$$
f\left(\beta_{i}^{\beta}\right): \exp \left(-\frac{1}{2 \sigma_{d B}^{2}}\left(\beta_{i}^{\circ}-A \log _{10} d-B-C \log _{10}\left(\frac{f_{c}}{5}\right)-X\right)^{2}\right)
$$

\section{A. CRLB for the case without uncertainty}

For the case without uncertainty in the frequency estimate, the unknown parameter vector $\boldsymbol{\theta}$ becomes $\boldsymbol{\theta}=\left[\begin{array}{ll}x & y\end{array}\right]^{T}$. Substituting (3) into $\ln f(P, \theta)$, gives:

$$
\ln f(\mathbf{P}, \boldsymbol{\theta})=\sum_{i=1}^{N} \ln f\left(\beta_{i}\right)
$$

Substituting(4)into (2), gives:

where

$$
J_{1}=E\left[\frac{\partial \ln f(\mathbf{P}, \boldsymbol{\theta})}{\partial \boldsymbol{\theta}}\left(\frac{\partial \ln f(\mathbf{P}, \boldsymbol{\theta})}{\partial \boldsymbol{\theta}}\right)^{T}\right]=\mathbf{H}_{1} \mathbf{Q}_{1}^{-1} \mathbf{H}_{1}^{T}
$$

$$
\mathbf{H}_{1}=\left[\begin{array}{ccc}
\frac{A}{d_{1} \ln 10} \frac{\partial d_{1}}{\partial x} & \mathrm{~L} & \frac{A}{d_{N} \ln 10} \frac{\partial d_{N}}{\partial x} \\
\frac{A}{d_{1} \ln 10} \frac{\partial d_{1}}{\partial y} & \mathrm{~L} & \frac{A}{d_{N} \ln 10} \frac{\partial d_{N}}{\partial y}
\end{array}\right], \mathbf{Q}_{1}=\operatorname{diag}\left\{\left[\begin{array}{lll}
\sigma_{d B}^{2} & \mathrm{~L} & \sigma_{d B}^{2}
\end{array}\right]\right\}, \frac{\partial d_{i}}{\partial x}=\frac{x-x_{i}}{d_{i}}
$$

The CRLB can be derived as $C R L B_{A}=\mathbf{J}_{1}^{-1}$.

\section{B. CRLB for the case with uncertainty}

It should be noted that Doppler shift and estimation errors will result in the uncertainty of carrier 
frequency estimation in a real situation. We model this uncertainty as a Gaussian random process based on the law of large numbers. For the case with uncertainty in the frequency estimate, the unknown parameter vector $\boldsymbol{\theta}$ becomes $\boldsymbol{\theta}=\left[\begin{array}{lll}x & y & f_{c}\end{array}\right]^{T}$. Assuming that $f_{c}^{\ell / c}$ is an estimate of $f_{c}$, the PDF of $f_{c} / \mathrm{c}$ can be written as:

$$
f(f / 0): \prod_{i=1}^{N} \exp \left(-\frac{1}{2 \sigma_{f_{c}}^{2}}\left(f f_{c i}-f_{c}\right)^{2}\right)
$$

According to the Bayes' theorem, we have:

$$
f(\mathbf{P}, \boldsymbol{\theta})=f(\mathbf{P} \mid \boldsymbol{\theta}) f(\boldsymbol{\theta})
$$

Substituting (3)and (6) into (7), gives:

$$
J_{2}=\mathbf{H}_{2} \mathbf{Q}_{1}^{-1} \mathbf{H}_{2}^{T}+\mathbf{Q}_{2}
$$

where

$$
\mathbf{H}_{2}=\left[\begin{array}{c}
\mathbf{H}_{1} \\
\frac{C}{f_{c} \ln 10} \mathbf{1}_{1 \times N}
\end{array}\right], \mathbf{Q}_{2}=\operatorname{diag}\left\{\left[\begin{array}{lll}
0 & 0 & N / \sigma_{f_{c}}^{2}
\end{array}\right]\right\}
$$

The CRLB can be derived as $C R L B_{B}=\left[\mathbf{J}_{2}^{-1}\right]_{2 \times 2}$.

\section{Performance comparison}

This subsection compares the performance between the above two cases through theoretical analysis. it can be derived that

$$
C R L B_{B}=\left[\mathbf{J}_{2}^{-1}\right]_{2 \times 2}=\mathbf{J}_{11}=\mathbf{H}_{1} \mathbf{Q}_{1}^{-1}\left(\mathbf{I}-K \mathbf{1}_{N \times N}\right) \mathbf{H}_{1}^{T}
$$

where

$$
K=\frac{\left(C /\left(f_{c} \ln 10\right)\right)^{2}}{\sigma_{d B}^{2}\left(\left(\frac{C}{f_{c} \ln 10}\right)^{2} \frac{N}{\sigma_{d B}^{2}}+\frac{N}{\sigma_{f_{c}}^{2}}\right)}=\frac{1}{N\left(1+\sigma_{d B}^{2} \delta\left(\sigma_{f_{c}}^{2}\right)\left(\frac{C}{f_{c} \ln 10}\right)^{2}\right)}
$$

It can be seen from WINNER II channel [5] that

$\sigma_{d B} \subset(1,10), \quad C \geq 20, f_{c}:(2,6), \quad N \sim(3,7)$.

$\sigma_{f_{c}}$ is the fluctuation of frequency caused by Doppler shift and estimation error, $\sigma_{f_{c}}$ is less than $1 \mathrm{MHz}$ generally. Thus $\sigma_{f_{c}} \leq 1 e 6 / 1 e 9=10^{-3}$. Substituting these parameters into (11), gives:

$$
K \sim 10^{-5},\left\|K \mathbf{1}_{N \times N}\right\|=\mathbf{I}
$$

Substituting (12) into (10), gives:

$$
C R L B_{B}=\mathbf{H}_{1} \mathbf{Q}_{1}^{-1}\left(\mathbf{I}-K \mathbf{1}_{N \times N}\right) \mathbf{H}_{1}^{T} \approx \mathbf{H}_{1} \mathbf{Q}_{1}^{-1} \mathbf{I} \mathbf{H}_{1}^{T}=\mathbf{H}_{1} \mathbf{Q}_{1}^{-1} \mathbf{H}_{1}^{T}=\mathbf{J}_{1}^{-1}=C R L B_{A}
$$

Equation (13) shows that the effects of the frequency uncertainty on the positioning accuracy can be negligible in a real situation.

\section{Simulation Results}

According to WINNER II channel model, the simulation parameters are set to be $\mathrm{A}=36.8$, $\mathrm{B}=43.8, \mathrm{C}=20, \mathrm{X}=0, \mathrm{~d}=100 \mathrm{~m}, f_{c}=2 \mathrm{GHz}$. Frequency uncertainty caused by Doppler shift and frequency estimation error is assumed to be zero-mean Gaussian process with variance $\sigma_{f_{c}}=10^{-3}$ in the units of GHz. The simulations are based on the cells of $2000 \mathrm{~m}$ of diameter. The coordinates of four BSs are BS $1(0,0) \mathrm{m}$ being the serving BS, BS $(1732,1000) \mathrm{m}, \mathrm{BS}_{3}(0,2000) \mathrm{m}$, and $\mathrm{BS}_{4}$ $(-1732,1000) \mathrm{m}$. The position of $\mathrm{MS}$ is uniformly distributed in the square space $-1000 \leq x, y \leq 1000 \mathrm{~m}$. The RMSEs and CRLBs are used to evaluate the impact of frequency 
change for positioning accuracy.

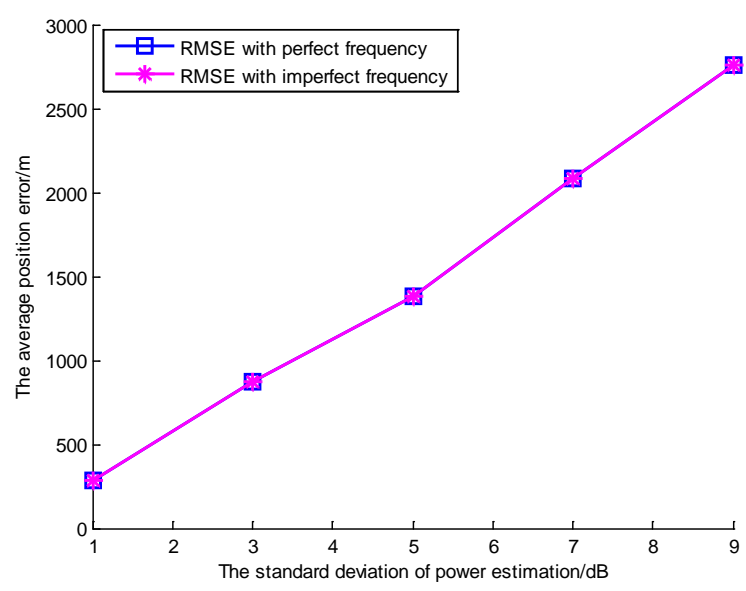

Fig.1. RMSEs comparisons using LS method

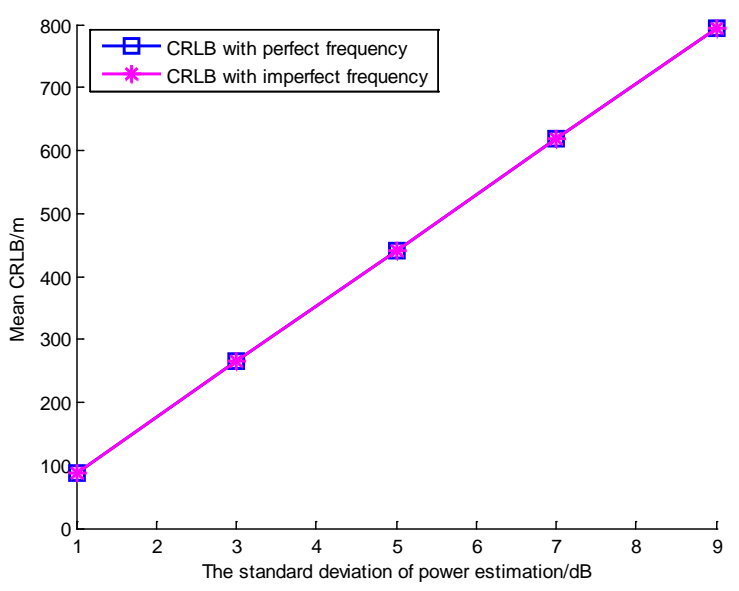

Fig.2.CRLBs comparisons

The simulations are performed to evaluate the impact of frequency uncertainty on positioning accuracy by RMSEs and CRLBs. From the above figures, it can be seen that RMSEs and CRLBs increase as the standard deviation of power estimation increase. It is observed from this simulation that RMSEs and CRLBs are the same in the two cases. In the other word, experimental results indicate that frequency uncertainty affects positioning accuracy so small that we can ignore it.

\section{Conclusions}

RSS based location method has attracted much attention because of its low complexity and cost of devices. Although many performance analysis have been addressed for RSS location method, the impact of frequency uncertainty on positioning accuracy for RSS has not been studied in the literature to the best of our knowledge. In this paper, theoretical analysis and simulations are both provided for analyzing the impact of frequency uncertainty on positioning accuracy. This paper evaluates the effects of frequency uncertainty on mobile location in terms of CRLB. Two CRLBs based WINNER II channel are derived for the cases with perfect or imperfect frequency estimation. Theoretical analysis and simulations show that the effects of the frequency uncertainty on performance of location estimation would be negligible.

\section{Acknowledgements}

This work was supported by the Open Research Fund of north engineering Co.,LTD of the electrification bureau group, and the Fundamental Research Funds for the Central Universities (ZYGX2013J026).

\section{References}

[1] N. Patwari, J. Ash, S. Kyperountas, A. Hero III, R. Moses, and N. Correal, "Locating the nodes: Cooperative localization in wireless sensor networks,” IEEE Signal Process. Mag., vol. 22, no. 4, pp. 54-69, July 2005

[2] Mohammad Kazemi, Behrad Mahboobi, and Mehrdad Ardebilipour, " Performance Analysis of Simultaneous Location and Power Estimation Using WLS Method for Cognitive Radio”, IEEE Communications Letters, Oct.2011,15(10),pp. 1062 - 1064

[3] Vaghefi, R.M., Gholami, M.R., Strom, E.G. "RSS-BASED SENSOR LOCALIZATION WITH UNKNOWN TRANSMIT POWER,” 2011 IEEE International Conference on Acoustics, Speech and Signal Processing (ICASSP), 2011, pp. 2480-2483

[4] Kay S. M., Fundamentals of Statistical Signal Processing: Estimation Theory. Englewood Cliffs, NJ: Prentice-Hall, 1993

[5] WINNER II D1.1.2, "WINNER II channel models,” https:// www.ist-winner.org/deliverables.html, September 2007. 